\title{
Factors influencing caregivers' uncertainty of children undergoing cardiac surgery in Bangkok, Thailand
}

\author{
Kunnara Maneekunwong, Arunrat Srichantaranit and \\ Wanlaya Thampanichawat \\ Department of Pediatric Nursing, Faculty of Nursing, Mahidol University, \\ Bangkok, Thailand
}

\begin{abstract}
Purpose - This study aims to determine the factors influencing caregivers' uncertainty about children undergoing cardiac surgery in a tertiary hospital in Bangkok, Thailand.

Design/methodology/approach - A correlational predictive study was conducted among 75 caregivers of children from infancy to fifteen years old who were undergoing first time cardiac surgery in a tertiary hospital in Bangkok, Thailand. Four questionnaires were inquired to evaluate caregivers' uncertainty about the illness, and influential factors included (1) perception of the severity of the illness, (2) credible authority of health-care providers and (3) information and emotional support. The descriptive statistics, Pearson correlation coefficient and multiple regression analysis were used to analyze the data and influential factors.

Findings - The significant influential factors affecting caregivers' uncertainty were (1) perception of the severity of the illness $(\beta=0.413, p<0.001)$, (2) credible authority of health-care providers $(\beta=-0.287$, $p=0.004)$ and (3) information and emotional support $(\beta=-0.223, p=0.026)$, and their explanation power was about $33.9 \%\left(R^{2}=0.339, F=13.630, p<0.001\right)$.

Research limitations/implications - A limitation of this study was that the researcher selected the sample group by convenient sampling and only caregivers of children who underwent cardiac surgery in Siriraj hospital were selected. Therefore, the sample group might have lacked variety and was not a good representative of the population. Future studies should be conducted by varying the setting and using randomized sampling.

Practical implications - This study provides clear recommendations to assess the perception of the severity of the illness by caregivers, build credibility and trust by providing quality care and should develop information and emotional support interventions for reducing the levels of caregivers' uncertainty of children undergoing cardiac surgery.

Originality/value - Health-care providers should develop the appropriate intervention for reducing caregivers' uncertainty by assessing the perception of the severity of the illness, providing information and emotional support and building trust for the caregivers of the children who are undergoing cardiac surgery.
\end{abstract}

Keywords Caregivers, Children, Congenital heart disease, Cardiac surgery, Thailand

Paper type Research paper

\section{Introduction}

Congenital heart disease (CHD) is a general term that describes a range of anatomical deformities of the heart and main blood vessels. The global incidence of CHD is approximately 9.4 cases per 1,000 live births [1]. Currently, in Thailand, it has been

(C) Kunnara Maneekunwong, Arunrat Srichantaranit and Wanlaya Thampanichawat. Published in Journal of Health Research. Published by Emerald Publishing Limited. This article is published under the Creative Commons Attribution (CC BY 4.0) licence. Anyone may reproduce, distribute, translate and create derivative works of this article (for both commercial and non-commercial purposes), subject to full attribution to the original publication and authors. The full terms of this licence may be seen at http:// creativecommons.org/licences/by/4.0/legalcode

Received 7 January 2021 Revised 12 March 2021 Accepted 10 May 2021 influencing caregivers' uncertainty

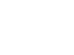


JHR

36,5

920

reported that each year 8,000 infants are born with some form of $\mathrm{CHD}$ amounting to 8 cases per 1,000 live births [2]. CHD is the main cause of death among children with congenital malformations [3] and can be classified into acyanotic $\mathrm{CHD}$ and cyanotic $\mathrm{CHD}$. These conditions are considered critical because the infant's survival depends on the cardiac function and relies on surgery as the main treatment method [4].

In Thailand, half the children with $\mathrm{CHD}$ require cardiac surgery each year [2]. According to the statistical records of Siriraj Hospital, 288 children had been treated by surgery, of which, $45 \%$ were acyanotic $\mathrm{CHD}$ patients and $55 \%$ were cyanotic $\mathrm{CHD}$ patients. However, some children had an extended period of surgery taking two years due to a lack of pediatric cardiothoracic surgeons [2]. On account of this situation, caregivers are more likely to experience stress and anxiety because they are aware that $\mathrm{CHD}$ is a serious illness and also know that cardiac surgery is a potentially life-threatening and devastating event [5]. Particularly in the cases of prolonged waiting time for cardiac surgery, caregivers experience a difficult time due to fear and uncertainty caused by observing unstable symptoms such as tachypnea, tachycardia or sudden cardiac arrest [6]. Caregivers of children undergoing the first cardiac surgery reportedly experienced uncertainty from the point of receiving the child's diagnosis until the day that their children underwent cardiac surgery, mainly due to the fear of intraoperative death or postoperative complications $[7,8]$. It therefore can be concluded that the timing prior to cardiac surgery is a critical time for caregivers as they experience feelings of uncertainty, stress or fear of losing their children from cardiac surgery.

Uncertainty can be defined as the inability to determine the meaning of illness-related events and is a cognitive state created when the individual cannot adequately structure or categorize an illness event because of insufficient cues [9]. Mishel developed four dimensions of uncertainty in parents caring for hospitalized children as follows: (1) Ambiguity refers to an inability of understanding the child's illness progression or treatment plan. The major source of ambiguity is the complexity of the device, medical technology or the child's unstable symptoms. (2) Lack of clarity is receiving incomplete explanations about the child's treatments, procedures or care system. For example, medical jargon used by health-care providers may lead caregivers to feel a lack of clarity. (3) Lack of information relevant to the child's disease and treatment - this occurs when the facts about an illness are unknown or not shared. (4) Unpredictability in this situation is an inability to predict the near future of the child's illness, outcomes of the treatment and the child's survival. Uncertainty in illness is influenced by the stimuli frame that includes symptom pattern, event familiarity and event congruence. In addition, cognitive capacity and structure providers, including social support and credible authorities in health-care providers also influence uncertainty [9]. Caregivers' uncertainty has been associated with higher levels of psychological distress such as stress, anxiety or depression [10] and this may develop behaviors similar to post-traumatic stress disorder (PTSD) such as insomnia, nervousness or fatigue [11] that in turn interferes with the caregiver's role and also the caregiver/child relationship [12]. A previous study showed that research regarding caregivers' uncertainty of children in their care with $\mathrm{CHD}$ remains underestimated. Limited studies conducted on caregivers' uncertainty mostly emphasized the postcardiac surgery period [13] providing a different aspect to precardiac surgery conditions. In order to prevent the negative impacts of caregivers' uncertainty of children undergoing cardiac surgery [10-12], this research aimed to study the factors influencing caregivers' uncertainty by using Mishel's uncertainty in illness concept as the theoretical framework [9].

Multiple factors influence a caregivers' uncertainty such as the perception of a child's illness severity [13, 14], information and emotional support received [15, 16], confidence in and accepted means of support from health-care providers [15, 17] or individual factors such as religion or education level. This study sets out to evaluate the caregivers' uncertainty of CHD 
and study predictive factors including (1) perception of the severity of the illness because the caregiver's perception of heart disease as a life-threatening disease as well as them facing the risk of losing a child due to cardiac surgery $[5,6]$ and their perception of the severity of the illness could influence their uncertainty. A study by Liengpun et al. [13] found that the perception of the severity of the illness was capable of predicting the parental uncertainty of children with acyanotic CHD $(\beta=0.217, p<0.05)$. (2) Information and emotional support influence the caregivers' uncertainty because the caregivers received information related to the operation procedures, risk or necessity of surgery as well as received emotional support such as sympathy from health-care providers. Such support helps caregivers to better understand the situation of the child's impending surgery [7]. This factor was supported by the study of Lin et al. [16] that found that information and emotional support can predict parental uncertainty of children with cancer $(\beta=-0.11, p<0.05)$. (3) Credible authority of health-care providers was the last factor that affects caregivers' uncertainty. This is because having confidence in the surgeon's competence, health-care providers and the hospital reputation can reduce caregivers' uncertainty and increase their confidence in the child's surgery outcome [7, 8]. Similarly, a study by Li and Lo [17] found that trust in health-care providers influenced parental uncertainty of children with autistic disorder $(\beta=-0.312, p<0.01)$.

To date, based on the literature reviews conducted in Thailand and overseas, there is no evidence of studying the extent of caregivers' uncertainty of children undergoing cardiac surgery in the preoperative period. There is value in researching this as the study findings would provide valuable information for the development of proper nursing programs to reduce uncertainty and the negative impact of uncertainty in the caregivers of children undergoing cardiac surgery.

\section{Methods}

Study design

The correlational predictive design was conducted to study the predictive capacity of the perception of the severity of the illness, information and emotional support, and credible authority of health-care providers on caregivers' uncertainty of children undergoing cardiac surgery.

\section{The population and sample group}

Potential participants were the caregivers of newborn to 15-year-old children expecting to undergo their first cardiac surgery in Siriraj Hospital, Bangkok, Thailand. The ages of the children receiving cardiac surgery varied from 0 to 15 years due to the type of $\mathrm{CHD}$, the severity of the heart disease, the nutritional and clinical status of the children [2] as well as the limitation of time for data collection due to the first researcher's academic commitments. The participants were selected by convenient sampling between February to July 2020. Inclusion criteria were (1) primary or immediate caregivers (e.g. mother, father or grandparent) who were providing care of the children since the diagnosis, (2) aged 18 to 59 years old, (3) able to understand and communicate in Thai and (4) no cognitive impairments.

\section{The sample sizes}

These were formulated to determine a sufficient sample size using the multiple regression formula. The effect size was specified from a previous study $[13,15]$ which was a medium effect size $\left(R^{2}=0.13\right)$. When substituted in the formula with an alpha 0.05 , power of test 0.8 , the required sample size was determined as 77 participants. After the six-month period, 75 participants were selected, which was lower than expected due to the coronavirus outbreak

\section{Factors influencing caregivers' uncertainty}


of 2019 (COVID -19) that led to limitations in accessing the sample group and also because some participants had their surgery dates postponed for a year. However, the normal distribution was tested by the Kolmogorov-Smirnov test with a result that it was a normal distribution that met the study assumption. Therefore, the sample size in this study was 75 participants.

A total of five instruments were used for data collection listed below:

(1) The demographic data form, developed by the researcher was used to collect the personal information of the children and caregivers such as age, gender, education level, family income, CHD type, previous admission history and waiting time.

(2) The Thai version of the Caregivers' Uncertainty of Children Undergoing Cardiac Surgery Scale was used to measure the perceived uncertainty regarding the illness for caregivers when confronting the prognosis of children's CHD and cardiac surgery. The instrument was modified from the Parent's Perception of Uncertainty Scale (PPUS) developed by Mishel [18] and translated into the Thai language by Suwannaosod [19]. This questionnaire comprised 31 items, each item was scored on a Likert-type scale from $1=$ strongly disagree to $5=$ strongly agree. The overall score, which ranged from 31 to 155 , with a higher score indicating greater uncertainty was categorized into three levels: low (31-72), moderate (73-114) and high (115-155).

(3) The Thai version of the Perception of the Severity of the Illness Scale was a singleitem scale developed by Mishel [18] and translated into the Thai language by Yotwongratsamee [20]. This scale was a rating scale where 1 determined a low perception of the child's illness severity and 10 determined a high perception of the child's illness severity.

(4) The Information and Emotional Support Scale was used to measure information and emotional support received from physicians and nurses and was developed by Poompan et al. [15]. This scale comprised 15 items with each item scored on a rating scale from $1=$ never to $5=$ always. The overall score ranged from 15 to 75 with a higher score indicating higher levels of information and emotional support received.

(5) The Credible Authority of Health-care Providers Scale was used to measure trustworthiness in the capability of the care received from physicians, nurses and the hospital's reputation. This instrument was developed by Poompan et al. [15]. The questions consisted of 4 items with each item scored on a five-point rating scale ( $1=$ strongly disagree to $5=$ strongly agree $)$ with scores ranging from $4-20$ points. The higher scores denoted higher credible authority of health-care providers.

\section{Quality of research instruments}

Instrument quality was tested by five qualified experts for content validity and accuracy. The questionnaire on caregivers' uncertainty in illness acquired a Content Validity Index (CVI) equal to 0.93 . The questionnaire on information and emotional support and credible authority of health-care providers acquired a CVI equal to 1 . The instrument's reliability was pilottested on 20 participants and was found to have acceptable internal consistency reliability with Cronbach's alpha coefficients of (1) 0.80 for the caregivers' uncertainty in illness, (2) 0.92 for the information and emotional support and (3) 0.80 for the credible authority of health-care providers. 
Ethical considerations

All research protocols and instruments were approved by the institutional review board of the Faculty of Medicine of Siriraj Hospital, Mahidol University. (COA no. Si 071/2020).
Factors influencing caregivers' uncertainty

\section{Data collection process}

The researcher introduced herself to the participants and informed each of them of the research objectives, data collection process, data protection rights of the participants and the benefits of the research project. After that, the participants were asked to read the participation sheet. Once the participants had expressed willingness to participate in the study, the researcher requested them to sign a consent form. Thereafter, the researcher explained to the participants how they were to answer the questionnaires which had a total of 51 questions and explained that they would require about 20-30 minutes to complete the questionnaire. After these explanations, the researcher allowed them to answer the questionnaires. Upon completion of the questionnaires, the researcher interviewed the participants using an open-ended question format regarding how they obtained information on children's cardiac surgery from physicians, nurses and other caregivers who had similar experiences. The interview time lasted about 10-15 min.

\section{Data analysis}

The data were analyzed by IBM SPSS software version 22. The demographic data of caregivers and children were analyzed by descriptive statistical analysis. The correlation of the variables was analyzed by the Pearson correlation coefficient. The predictive capacity of perception of the severity of illness, information and emotional support, and credible authority of health-care providers on caregivers' uncertainty was analyzed by multiple regression analysis.

\section{Results}

\section{Caregivers' characteristics}

Results of the characteristics of 75 caregivers in this study showed that $86.7 \%$ of principal caregivers were mothers aged between $20-54$ years old with an average age of 31.3 years $(\mathrm{SD}=6.78)$. The majority of participants $(56 \%)$ had completed their studies up to the secondary level, followed by $32 \%$ who had completed a bachelor's degree as shown in Table 1.

\section{Children's characteristics}

The age of the children was between 10 days and 13 years with an average age of 1 year 8 months $(S D=2.72)$. The majority $(61.3 \%)$ were infants and $38.7 \%$ were between 1 year and 13 years. Children who had been diagnosed with acyanotic $\mathrm{CHD}$ totaled $49.3 \%$, and the percentage diagnosed with cyanotic $\mathrm{CHD}$ was $50.7 \%$. The majority of children $(76 \%)$ received total corrective surgery while $24 \%$ received palliative surgery. The waiting time for cardiac surgery was an average of 4.6 months $(S D=6.32)$. A total of $73.3 \%$ had no hospitalization history due to CHD complications as shown in Table 2.

\section{The score of variables}

Caregivers had a moderate level of uncertainty about the illness score across four dimensions. For the perception of the severity of the illness, information and emotional support, and credible authority of health-care providers, caregivers scored high as shown in Table 3. 
Relationship with children

Father

Mother

8

65

10.7

Grandparent

Age (years)

$\mathbf{9 2 4} \quad \begin{array}{ll}\text { Age } \\ 18-29\end{array}$

30-39

$40-49$

$50-59(M=31.3, S D=6.78$, range $=20-54)$

$\begin{array}{rc}30 & 40 \\ 36 & 48 \\ 7 & 9.3 \\ 2 & 2.7\end{array}$

\section{Education}

Primary education

Table 1.

Characteristics of caregivers

\section{The correlation of the variables}

The analysis of the correlation of the variables by Pearson's correlation coefficient showed that the variables with statistically significant correlation with caregivers' uncertainty included (1) the perception of the severity of the illness $(r=0.47, p<0.01)$, (2) information and emotional support $(r=-0.36, p<0.01)$ and (3) credible authority of health-care providers $(r=-0.31, p<0.01)$, Table 4.

\section{The factors influencing caregivers' uncertainty about the illness}

The analysis of predictive power by multiple regression analysis found that the perception of the severity of the illness, credible authority of health-care providers, information and emotional support were capable of predicting the caregivers' uncertainty of children undergoing cardiac surgery with $33.9 \%$ statistical significance (adjusted $R^{2}=0.339$, $F=13.630, p<0.001$ ). The factors with the predictive capability of the caregivers' uncertainty with statistical significance included (1) the perception of the severity of the illness (2) credible authority of health-care providers (3) information and emotional support as shown in Table 5.

\section{Discussion}

The results demonstrated that three variables including (1) the perception of the severity of the illness (2) credible authority of health-care providers and (3) information and emotional support were significant predictors of caregivers' uncertainty while the children with $\mathrm{CHD}$ were undergoing cardiac surgery. These findings are congruent with previous studies.

Perceptions of the severity of the illness are factors that have a statistically significant influence on the caregivers' uncertainty about the illness $(\beta=0.413, p<0.001)$. It can be explained that children with CHDs who undergo cardiac surgery often have complex heart deformities and most CHDs are potentially life-threatening. One- third of these had a history of hospitalization from complications of $\mathrm{CHD}$ such as pneumonia, heart failure or anoxic spells. Some children with $\mathrm{CHD}$ may require more intensive treatment, and caregivers often sacrifice a lot of their time and expend energy to care for the child with $\mathrm{CHD}$ at the cost of their own physical and emotional needs that could lead to stress, anxiety and uncertainty $[5,6]$. On the surgery day, in particular, caregivers perceived this period as a critical period $[5,8]$ due to 
the risks involved in the surgery, the complexity of the treatments as well as long-term issues including the potential for life-long physical limitations [8]. Therefore, the caregivers perceived the high level of children's illness severity with some ambivalence regarding the child's survival. This finding is supported by the findings of Madeo et al. [14] who found that parents who perceived the child's illness to be worse, experienced more uncertainty.

Credible authority of health-care providers was another statistically significant factor that influenced the caregivers' uncertainty $(\beta=-0.287, p=0.004)$. One explanation is that
Factors influencing caregivers' uncertainty

\begin{tabular}{|c|c|c|}
\hline Characteristics & Frequency & Percentag \\
\hline \multicolumn{3}{|l|}{ Gender } \\
\hline Male & 44 & 58.6 \\
\hline Female & 31 & 41.4 \\
\hline \multicolumn{3}{|l|}{ Age (years) } \\
\hline $0-1$ & 46 & 61.3 \\
\hline$>1-3$ & 14 & 18.6 \\
\hline$>3-6$ & 10 & 13.4 \\
\hline$>6-13(M=1.86, \mathrm{Sd}=2.72$, range $=0.03-13.18)$ & 5 & 6.7 \\
\hline \multicolumn{3}{|l|}{ Diagnosis } \\
\hline \multicolumn{3}{|l|}{ Acyanotic congenital heart disease $(N=37)$} \\
\hline VSD & 16 & 21.3 \\
\hline VSD with ASD/ large PDA/ Moderate MR/ CoA & 13 & 17.3 \\
\hline AVSD & 3 & 4 \\
\hline Others such as ASD, CoA, mild AS with mild AR, and severe AR with severe MR & 5 & 6.6 \\
\hline \multicolumn{3}{|l|}{ Cyanotic congenital heart disease $(N=38)$} \\
\hline TGA with VSD/ ASD/ PDA & 10 & 13.3 \\
\hline TOF & 7 & 9.3 \\
\hline UVH/HLHS & 4 & 5.4 \\
\hline TAPVR & 4 & 5.4 \\
\hline DORV with PDA & 3 & 4 \\
\hline $\begin{array}{l}\text { Others such as Dextrocardia, IAA, Truncus arteriosus, PA with IVS, Isomerism } \\
\text { and TA with VSD/PA/PDA }\end{array}$ & 10 & 13.4 \\
\hline \multicolumn{3}{|l|}{ Cardiac surgery } \\
\hline Total correction surgery & 57 & 76 \\
\hline Palliative surgery & 18 & 24 \\
\hline \multicolumn{3}{|l|}{ Waiting time (months) } \\
\hline$\leq 3$ & 42 & 56 \\
\hline$>3(M=4.60, S D=6.32$, range $=0.23-36.50)$ & 33 & 44 \\
\hline \multicolumn{3}{|c|}{ History of hospitalization from complications of CHD (pneumonia, congestive heart failure, anoxic spells) } \\
\hline No & 55 & 73.3 \\
\hline Yes & 20 & 26.7 \\
\hline \multicolumn{3}{|l|}{ Type of ward before surgery } \\
\hline Pediatric inpatient unit & 51 & 68 \\
\hline Intensive care unit & 24 & 32 \\
\hline
\end{tabular}

Note(s): VSD = Ventricular septal defect, ASD = Atrial septal defect, PDA = Patent ductus arteriosus, $\mathrm{MR}=$ Mitral regurgitation, $\mathrm{CoA}=$ Coarctation of the Aorta, AVSD = Atrioventricular septal defect, $\mathrm{AS}=$ Aortic stenosis, $\mathrm{AR}=$ Aortic regurgitation, $\mathrm{TGA}=$ Transposition of the great arteries, $\mathrm{PA}=$ Pulmonic atresia, TOF $=$ Tetralogy of Fallot, UVH = Univentricular heart, HLHS = Hypoplastic left heart syndrome, TAPVR = Total anomalous pulmonary venous return, DORV $=$ Double outlet of right ventricle, IAA = Interrupted aortic arch, IVS = Intact ventricular septum, TA = Tricuspid atresia

Table 2. Characteristics of the children 
JHR

36,5

926

Table 3.

The score of variables

children with $\mathrm{CHD}$ received treatment and care by a multidisciplinary team in Siriraj Hospital, which is the country's largest tertiary and quaternary care medical center. The highly experienced pediatric cardiologists, pediatric cardiac care nurses and surgeons shared responsibilities for providing comprehensive medical treatment and services. Statistics of cardiac surgery in children compiled by the society of thoracic surgeons of Thailand in 2019 found that Siriraj Hospital was ranked number 1 for cardiac surgery in children [21]. This may result in the caregivers having more trust and confidence in the upcoming surgery, and that reduced their uncertainty. The result is consistent with the study of Poompan et al. [15] on the parents of children during a critical illness period in Siriraj Hospital. The result found that credible authority of health-care providers negatively related to parental uncertainty.

Information and emotional support were the last factors that statistically significantly influenced the caregivers' uncertainty $(\beta=-0.223, p=0.026)$. It can be explained that sufficient information about cardiac surgery including the cardiac structure, cardiac surgery procedure, pathology and the care pathway from health-care providers could increase caregivers' understanding due to this information meeting the caregivers' information needs [22]. A better understanding of children's cardiac surgery might change the caregiver's perception of their children's cardiac surgery more positively [23]. Regarding emotional support, surgeons and nurses played a major role in providing emotional support such as encouragement or confidence in caring for children during admission. The emotional support

\begin{tabular}{lccrll}
\hline Variables & Possible range & Actual range & $M$ & SD & Interpretation \\
\hline Uncertainty about the illness & & & & & \\
Total scores & $31-155$ & $77-119$ & 95.14 & 7.63 & Moderate \\
Ambiguity & $13-65$ & $33-57$ & 44.78 & 4.86 & Moderate \\
Lack of clarity & $9-45$ & $18-30$ & 23.23 & 2.38 & Moderate \\
Lack of information & $5-25$ & $9-18$ & 13.56 & 2.12 & Moderate \\
Unpredictability & $4-20$ & $7-18$ & 13.18 & 2.32 & Moderate \\
Perception of the severity of the illness & $1-10$ & $5-10$ & 7.92 & 1.22 & High \\
Information and emotional support & $15-75$ & $52-71$ & 63.26 & 4.12 & High \\
Credible authority of health-care providers & $4-20$ & $14-20$ & 17.36 & 1.68 & High \\
\hline
\end{tabular}

\begin{tabular}{lcccr}
\hline Variables & 1 & 2 & 3 & 4 \\
\hline 1. Perception of the severity of the illness & 1 & & & \\
2. Information and emotional support & $-0.24^{*}$ & 1 & & \\
3. Credible authority of health-care providers & 0.01 & 0.12 & 1 & \\
4. Caregivers' uncertainty in illness & $0.47^{* *}$ & $-0.36^{* *}$ & $-0.31^{* *}$ & 1 \\
Note(s): ${ }^{* * *} p<0.01,{ }^{*} p<0.05$ & & & & \\
\hline
\end{tabular}

Table 4.

Correlation of the variables

\begin{tabular}{lrrrrr}
\hline Variables & $B$ & SE & $\beta$ & $t$ & $p$-value \\
\hline 1. Perception of the severity of the illness & 2.555 & 0.587 & 0.413 & 4.229 & $<0.001$ \\
2. Credible authority of health-care providers & -1.468 & 0.414 & -0.287 & -3.008 & 0.004 \\
3. Information and emotional support & -0.382 & 0.173 & -0.223 & -2.271 & 0.026 \\
Note(s): $*$ Statistically significant at $p \leq 0.05$; constant & $=123.388, R^{2}=0.365$, adjusted $R^{2}=0.339$, \\
$F=13.630, p<0.001$ & & & & \\
\end{tabular}


gained provided the caregiver with more emotional stability and helped them to perceive and understand the information concerning children's cardiac surgery. Moreover, caregivers had received adequate information and support throughout in a friendly environment and had been provided with telephone contact details to consult the experienced health-care providers online. Therefore, the uncertainty about the illness greatly subsided [9]. The result was consistent with the study in parents of children with cancer [16] which found that social support can directly influence parental uncertainty by providing information or affective support and thus reduce the uncertainty regarding the illness.

\section{Conclusions}

The findings from this study contribute to the body of knowledge regarding the uncertainty among caregivers of children undergoing cardiac surgery. Caregivers who perceive their child's illness to be more severe, tend to perceive greater uncertainty. It is also true that when parents receive less information and emotional support from health-care providers and feel less credible authority of health-care providers, the parents perceive more uncertainty regarding their children's illness. Based on the results of this study, we recommend that health-care providers assess the perception of the severity of the illness in caregivers, build credibility and trust by providing quality care, and develop information and emotional support interventions for reducing the levels of caregivers' uncertainty regarding their children undergoing cardiac surgery.

\section{Recommendations}

The results of the study should be incorporated into the design of an experimental research study about a nursing program to reduce the uncertainty about the illness among caregivers of children undergoing cardiac surgery.

\section{Limitation of the study}

A limitation of this study was that the researchers selected the participants via convenience sampling and only caregivers of children who underwent cardiac surgery at Siriraj Hospital were selected. Therefore, the sample group may lack diversity and may not be a good representative of the general population. Future studies should be conducted in various settings and should employ random sampling.

Conflicts of Interest: None

\section{References}

1. Liu Y, Chen S, Zuhlke L, Black GC, Choy MK, Li N, et al. Global birth prevalence of congenital heart defects 1970-2017: updated systematic review and meta-analysis of 260 studies. Int J Epidemiol. 2019; 48(2): 455-63. doi: 10.1093/ije/dyz009.

2. Pongpanich B. Pediatric congenital heart disease in Thailand. In: Sirijongkolthong B, Panamontha M, Durongpisitkul K, Kengsakun U, Vijarnsorn C, Witessonthi K, et al., (Eds). Pediatric cardiology. 1st ed. Bangkok: I Group Press; 2012: 1-4. (in Thai).

3. GBD 2017 Congenital Heart Disease Collaborators. Global, regional, and national burden of congenital heart disease, 1990-2017: a systematic analysis for the Global Burden of Disease Study 2017. Lancet Child Adolesc Health. 2020; 4(3): 185-200. doi: 10.1016/S2352-4642(19)30402-X.

4. Holst KA, Said SM, Nelson TJ, Cannon BC, Dearani JA. Current interventional and surgical management of congenital heart disease: specific focus on valvular disease and cardiac arrhythmias. Circ Res. 2017; 120(6): 1027-44. doi: 10.1161/CIRCRESAHA.117.309186.

5. Sjostrom-Strand A, Terp K. Parents' experiences of having a baby with a congenital heart defect and the child's heart surgery. Compr Child Adolesc Nurs. 2019; 42(1): 10-23. doi: 10.1080/24694193.2017.1342104. 
JHR

36,5

928
6. Srichantaranit A, Chontawan R. Caring for infants with congenital heart disease prior to cardiac surgery: the impacts on families. J Nurs Sci. 2011; 29(Suppl.2): 7-18.

7. Srichantaranit A, Thampanichawat W. Mothers' experience of having their children undergoing cardiac surgery: a qualitative study. Thai J Nurs Counc. 2018; 33(1): 74-88.

8. Nakazuru A, Sato N, Nakamura N. Stress and coping in Japanese mothers whose infants required congenital heart disease surgery. Int J Nurs Pract. 2017; 23(Suppl.1): e12550. doi: 10.1111/ijn.12550.

9. Mishel MH. Uncertainty in illness. Image J Nurs Sch. 1988; 20(4): 225-32.

10. Szulczewski L, Mullins LL, Bidwell SL, Eddington AR, Pai ALH. Meta-analysis: caregiver and youth uncertainty in pediatric chronic illness. J Pediatr Psychol. 2017; 42(4): 395-421. doi: 10.1093/ jpepsy/jsw097.

11. Franck LS, Wray J, Gay C, Dearmun AK, Lee K, Cooper BA. Predictors of parent post-traumatic stress symptoms after child hospitalization on general pediatric wards: a prospective cohort study. Int J Nurs Stud. 2015; 52(1): 10-21. doi: 10.1016/j.ijnurstu.2014.06.011.

12. Malin KJ, Johnson TS. A concept analysis of parental uncertainty in illness of an infant. MCN Am J Matern Child Nurs. 2019; 44(4): 206-11. doi: 10.1097/nmc.0000000000000535.

13. Liengpun N, Teerarungsikul N, Sananreangsak S. Predictors of uncertainty among parents of children with acyanotic congenital heart disease. J Nurs Sci Chulalongkorn Univ. 2017; 29(2): 76-86. (in Thai).

14. Madeo AC, O’Brien KE, Bernhardt BA, Biesecker BB. Factors associated with perceived uncertainty among parents of children with undiagnosed medical conditions. Am J Med Genet A. 2012; 158A(8): 1877-84. doi: 10.1002/ajmg.a.35425.

15. Poompan P, Tilokslulchai F, Prasopkittikun T, Limprayoon K. Factors influencing parents' uncertainty in chronically ill children with intubation during critical period. J Nurs Sci. 2012; 30(3): 15-24. (in Thai).

16. Lin L, Yeh CH, Mishel MH. Evaluation of a conceptual model based on Mishel's theories of uncertainty in illness in a sample of Taiwanese parents of children with cancer: a cross-sectional questionnaire survey. Int J Nurs Stud. 2010; 47(12): 1510-24. doi: 10.1016/j.ijnurstu.2010.05.009.

17. Li TY, Lo JL. The predictors of uncertainty in mothers of children with autism spectrum disorder. Child Health Care. 2016; 45(2): 147-64. doi: 10.1080/02739615.2014.979924.

18. Mishel MH. Parents' perception of uncertainty concerning their hospitalized child. Nurs Res. 1983; 32(6): 324-30.

19. Suwannaosod S. Uncertainty, social support, and coping strategies among parents of children with cancer. Chiang Mai: Chiang Mai University; 2004.

20. Yotwongratsamee R. Factors influencing uncertainty in illness of parents with children admitted in intensive care unit. Bangkok: Mahidol University; 2006.

21. The Society of Thoracic Surgeons of Thailand. Statistics of heart surgery in Thailand in 2019. [cited 2020 Oct 1]. Available at: http://thaists.org/news_files/news_file_672.pdf.

22. Aranha PR, Dsouza SN. Preoperative information needs of parents: a descriptive survey. J Res Nurs. 2019; 24(5): 305-14. doi: 10.1177/1744987118821708.

23. Vainberg L, Vardi A, Jacoby R. The Experiences of parents of children undergoing surgery for congenital heart defects: a holistic model of care. Front Psychol. 2019; 10: 2666. doi: 10.3389/fpsyg. 2019.02666 .

\section{Corresponding author}

Arunrat Srichantaranit can be contacted at: arunrat.sri@mahidol.ac.th

For instructions on how to order reprints of this article, please visit our website:

www.emeraldgrouppublishing.com/licensing/reprints.htm

Or contact us for further details: permissions@emeraldinsight.com 